University of Nebraska - Lincoln

DigitalCommons@University of Nebraska - Lincoln

Biochemistry -- Faculty Publications

Biochemistry, Department of

1999

Early and late endosomal compartments of Entamoeba histolytica are enriched in cysteine proteases, acid phosphatase and several Ras-related Rab GTPases

\author{
Lesly A. Temesvari \\ Feist-Weiller Cancer Center, Shreveport, LA \\ Ed Harris \\ University of Nebraska - Lincoln, eharris5@unl.edu \\ Samuel L. Stanley Jr. \\ Washington University School of Medicine, St. Louis, \\ James Cardelli \\ LSU Health Sciences Center, Shreveport, LA, jcarde@lsuhsc.edu
}

Follow this and additional works at: https://digitalcommons.unl.edu/biochemfacpub

Part of the Biochemistry, Biophysics, and Structural Biology Commons

Temesvari, Lesly A.; Harris, Ed; Stanley, Samuel L. Jr.; and Cardelli, James, "Early and late endosomal compartments of Entamoeba histolytica are enriched in cysteine proteases, acid phosphatase and several Ras-related Rab GTPases" (1999). Biochemistry -- Faculty Publications. 52.

https://digitalcommons.unl.edu/biochemfacpub/52

This Article is brought to you for free and open access by the Biochemistry, Department of at DigitalCommons@University of Nebraska - Lincoln. It has been accepted for inclusion in Biochemistry -- Faculty Publications by an authorized administrator of DigitalCommons@University of Nebraska - Lincoln. 


\title{
Early and late endosomal compartments of Entamoeba histolytica are enriched in cysteine proteases, acid phosphatase and several Ras-related Rab GTPases
}

\author{
Lesly A. Temesvari, ${ }^{1}$ Edward N. Harris, ${ }^{2}$ Samuel L. Stanley Jr., ${ }^{3}$ James A. Cardelli ${ }^{1,2}$ \\ 1. Feist-Weiller Cancer Center, Shreveport, LA 71130, USA \\ 2. Department of Microbiology and Immunology, \\ Louisiana State University Medical Center, Shreveport, LA 71130, USA \\ 3. Departments of Medicine and Molecular Microbiology, \\ Washington University School of Medicine, St. Louis, MO 63110, USA \\ Corresponding author - L. A. Temesvari, present address: Department of Biological Sciences, \\ Clemson University, Clemson, SC 29634-1903, USA. Tel.: + 1-864-6562328.
}

\begin{abstract}
Pure populations of early and late endosomes of Entamoeba histolytica were isolated by magnetic fractionation and characterized. It was shown that these vesicles were enriched in acid phosphatase and cysteine protease activities. An important virulence factor, a 27-kDa cysteine protease, was also enriched in early and late endosomes of E. histolytica. These data suggest that E. histolytica hydrolases reside in compartments that are part of or communicate with the endosomal pathway. To begin to identify the role of Rab GTPases in E. histolytica, an oligonucleotide approach was employed to screen an E. histolytica cDNA library for genes encoding Rab-like proteins. cDNAs encoding a Rab 11-like protein (EhRab11) and a novel Rab protein (EhRabA) were isolated and characterized. The EhRab11 cDNA predicts a polypeptide of at least 206 amino acids with a molecular mass of at least $23.2 \mathrm{kDa}$. Phylogenetic analysis and alignment of EhRab11 with other Rab proteins demonstrated that EhRab11 shared significant homology at the amino acid level with Rab 11-like proteins from a number of other eukaryotes, suggesting that EhRab11 is a Rab11 homolog for E. histolytica. The EhRabA clone predicts a polypeptide of 219 amino acids with a molecular mass of at least $24.5 \mathrm{kDa}$. EhRabA shared only limited homology at the amino acid level with other Rab proteins, suggesting that it is a novel member of this family of GTP-binding proteins. Finally, Western blot analysis demonstrated that EhRab11 and a previously described Rab7-like GTPase from E. histolytica was enriched in magnetically purified endosomal compartments of this organism.
\end{abstract}

Keywords: acid phosphatase, cysteine protease, endosome, Entamoeba histolytica, Rab GTPase

\section{Introduction}

The protozoan parasite, Entamoeba histolytica, infects $10 \%$ of the world population, leading to 50 mil- lion cases of invasive amebiasis and 100,000 deaths annually [1]. Although improvements in sanitation in affected areas would prevent many infections with E. histolytica, modest advances in this respect 
demand that improved methods of disease diagnosis, treatment and prevention be investigated.

Infection is acquired by ingestion of the cyst form of the parasite [2]. Excystation occurs in the small intestine and is characterized by the release of amoeboid trophozoites, which move to and colonize the bowel lumen. In the bowel lumen bacteria, erythrocytes, and cell debris serve as the major food source. Uptake of these nutrients is achieved by endocytic processes (pinocytosis, phagocytosis) and digestion of the internalized macromolecules is likely to be carried out by hydrolytic enzymes, including proteases [3], which may reside in endo-lysosomal-like compartments.

Endocytic processes are poorly defined in E. histolytica. It is known, however, that these amoebae can actively phagocytose large particles such as bacteria and erythrocytes, and the ability to phagocytose bacteria appears to be correlated with virulence $[4,5]$. In addition, this organism exhibits robust fluid phase pinocytosis, turning over $30 \%$ of its volume per hour $[6,7]$. There are two pinocytic compartments in E. histolytica [6]. The first (early) compartment consists of non-acidic, large vesicles ( $>2 \mu \mathrm{m}$ in diameter) that rapidly exchange their contents with the external milieu. Trafficking of fluid phase to and from this compartment accounts for most of the pinocytosis carried out by $E$. histolytica. The second (late) compartment consists of smaller vesicles with acidified contents [6]. The physiological relevance of these two compartments has not been discerned.

Following depletion of the host protective mucous blanket, amoebae can lyse colonic epithelial cells and invade the bowel mucosa to cause symptomatic infection characterized by massive and sometimes fatal destruction of host tissue [8]. The potent cytolytic capabilities of E. histolytica have been attributed to a family of pore-forming peptides, termed amoebapores [9], and hydrolytic enzymes. One group of the hydrolytic enzymes implicated in E. histolytica pathogenicity is the cysteine proteases, which are constitutively secreted from E. histolytica trophozoites [3, 10]. Cysteine proteases, ranging in molecular weights of 16-116 kDa, have been identified in extracts of Entamoeba [3]. In particular, a 27-kDa cysteine proteinase has been shown to participate in adherence [11] of amoebae to host cell extracellular matrix and in amoebic liver abscess formation [11, 12]. Another hydrolytic enzyme, acid phosphatase, has also been implicated in host cell destruction [13, 14]. Despite the importance of the E. histolytica endo-lysosomal and secretory systems in nutrition and invasion of the organism, relatively little is known about the molecular factors governing these systems including the associated proteins which may regulate endocytic or secretory function.

In other eukaryotes, the Rab family of small molecular weight Ras-like GTPases play important roles in the regulation of vesicle trafficking between various compartments along endo-lysosomal and secretory pathways $[15,16]$. They also serve as useful markers along these pathways as the cytoplasmic faces of each compartment are often associated with a unique Rab protein or set of Rab proteins. One of the unique structural features of Rabs is the carboxy terminal amino acid sequence, which may be characterized by a "-cysteine-cysteine" or a "cysteine-X-cysteine" motif [17]. The carboxy terminal cysteines are post-translationally modified by geranylgeranyl groups [18] which facilitate membrane interaction.

Rabs can cycle between active (GTP-bound) and inactive (GDP-bound) states; this switching is controlled, in part, by the transient association of the Rab protein with regulatory proteins [16]. By alternating between membrane-bound and cytosolic forms and by binding and hydrolyzing GTP, Rab GTPases can function as "molecular switches" to regulate vesicle trafficking; a process by which material is transported throughout the cell and by which the uniqueness of each endocytic or secretory compartment is maintained. Given the proposed role of Rab proteins in other eukaryotic cells, it is likely that Rab-like GTPases regulate similar processes in E. histolytica. To date, only one Rab, a Rab7-related GTPase, has been identified in this organism [19], and its function has not been discerned.

To further our understanding of the endosomal system of E. histolytica we have isolated pure pop- 
ulations of early and late endosomes by magnetic fractionation and characterized their associated proteins. In particular, we demonstrate that these compartments are enriched in acid phosphatase activity and cysteine proteases including the 27-kDa cysteine protease. In addition, to begin to identify the role of Rab GTPases in E. histolyt$i c a$, we have used an oligonucleotide approach to screen an E. histolytica cDNA library for genes encoding additional Rab-like proteins. We report the isolation and sequence analysis of cDNAs encoding a Rab11-like species (EhRab11) and a novel Rab protein (EhRabA) from E. histolytica. We further demonstrate that EhRab11 and the previously described Rab7-like GTPase is enriched in endosomal compartments of E. histolytica.

\section{Materials and methods}

\subsection{Generation of iron-dextran particles}

Iron-dextran particles were generated according to the method of Rodriguez-Paris et al. [20].

\subsection{Strains and culture conditions}

E. histolytica trophozoites (strain HM-1:IMSS) were cultured axenically in TYI-S-33 media [21] in screw cap tubes at $37^{\circ} \mathrm{C}$.

\subsection{Effect of iron-dextran on growth and pinocytosis}

To measure the effect of iron-dextran on growth and pinocytosis, cells were incubated with fluorescein isothiocyanate (FITC)-dextran (70,000 $M_{\mathrm{r}^{\prime}}$; Sigma, St. Louis, MO; $2 \mathrm{mg} / \mathrm{ml}$ ) in TYI-S-33 [21] medium in the presence or absence of iron-dextran $(2 \mathrm{mg} / \mathrm{ml})$. At the time points indicated (Section $3)$, the cells were detached by chilling on ice for 10 min, decanted, pelleted by centrifugation at $500 \times$ $g$ at $4^{\circ} \mathrm{C}$, washed twice in cold Buffer $\mathrm{H}$ (5 mM glycine, $100 \mathrm{mM}$ sucrose, $\mathrm{pH}$ 8.5) and resuspended in wash buffer. The cells were then counted by microscopic visualization using a Bright Line Hema- cytometer (Reichert, Buffalo, NY). In addition, the cells were lysed in 10\% (v/v) Triton X-100 and the intracellular fluorescence was measured with a Hitachi Fluorospectrophotometer (Model F-4010) using excitation and emission wavelengths of 492 and $525 \mathrm{~nm}$, respectively. Fluorescence was converted to volume using a standard curve of FITC. In addition, cells were lysed as described above and the cell-associated iron was measured as described [22].

\subsection{Measurement of the intra-lumenal $\mathrm{pH}$ of late endosomes}

Endosomal $\mathrm{pH}$ in living E. histolytica trophozoites was measured by a dual excitation ratio method with FITC-dextran as a $\mathrm{pH}$ probe as described [23]. Briefly, late endosomes of trophozoites were labeled with FITC-dextran $(2 \mathrm{mg} / \mathrm{ml}$; see Section 3 for optimal loading regime). At the times indicated, cells were harvested as described above, washed with and resuspended in $50 \mathrm{mM}$ MES buffer $(\mathrm{pH}$ 6.5) at a concentration of $3 \times 10^{6}$ cells $/ \mathrm{ml}$. The cells were diluted $20 \times$ in MES buffer and fluorescence at 520 $\mathrm{nm}$ following excitation at 450 and $495 \mathrm{~nm}$ was measured. The fluorescence excitation ratio at 495 and $450 \mathrm{~nm}$ (value using excitation wavelength of 495 $\mathrm{nm}$, value using excitation wavelength of $450 \mathrm{~nm}$ ) was calculated, and endosomal $\mathrm{pH}$ was determined from an in vitro standard curve of FITC-dextran in a $\mathrm{pH}$ range of 4-7.

\subsection{Magnetic purification of early and late endosomes}

For magnetic fractionation, E. histolytica trophozoites were grown in $100 \mathrm{ml}$ of axenic medium, TYI-S-33 [21], in sealed tissue culture flasks (T-175), at $37^{\circ} \mathrm{C}$ in a $\mathrm{CO}_{2}$ incubator. Typically six to eight flasks were used per experiment which represented a total of 4.8-6.4 $\times 10^{8}$ cells. Early or late compartments were labeled with $2 \mathrm{mg} / \mathrm{ml}$ iron-dextran as described in Section 3. After labeling, the cells were harvested as described above, washed twice with cold TYI-S-33, and resuspended at a concentration of $2 \times 10^{8}$ cells $/ \mathrm{ml}$ in Buffer $\mathrm{H}$. The amoebae 
were homogenized by passage through an $8-\mu \mathrm{m}$ polycarbonate filter (Poretics, Livermore, CA). In all instances, except when cysteine protease activity was measured, the homogenate was supplemented with a protease inhibitor cocktail consisting of $1 \mathrm{mM}$ ethylenediaminetetraacetic acid (EDTA), $1 \mathrm{mM}$ ethylene bis(oxyethylenenitrilo)tetraacetic acid (EGTA), $10 \mu \mathrm{g} / \mathrm{ml}$ leupeptin, $20 \mu \mathrm{g} /$ $\mathrm{ml}$ chymostatin, and $20 \mu \mathrm{g} / \mathrm{ml}$ pepstatin. Iron-containing endosomal compartments were purified from the homogenate by magnetic fractionation as described [20, 24].

Briefly, a column containing a loose mat of fine

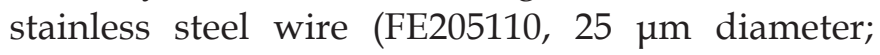
Goodfellow Corp., Malvern, PA) was mounted between the pole faces of a laboratory electromagnet operating at 0.8 tesla. The homogenate was pumped into the column at $90 \mathrm{ml} / \mathrm{h}$. Unretained effluent flowed through the column and the column was washed extensively with at least ten column volumes of Buffer $\mathrm{H}$. The retained fraction was retrieved by shutting off the magnet and forcing 2 column volumes of Buffer $\mathrm{H}$ up through the wire mesh. The retained vesicles were aspirated with a Pasteur pipette and were concentrated by centrifugation at $39,000 \times \mathrm{g}$ for $30 \mathrm{~min}$ at $4^{\circ} \mathrm{C}$. Vesicle-associated iron was measured as described [22]. Purified vesicles were characterized with respect to enrichment of hydrolases and Rab GTPases as described below.

\subsection{Acid phosphatase activity}

Whole cells, purified early and late vesicles and column flow-through fractions were lysed by the addition of Triton X-100 to a final concentration of $0.5 \%(\mathrm{v} / \mathrm{v})$. The activity of acid phosphatase in the fractions was determined as described [25].

\subsection{Measurement of cysteine protease activity}

Proteinases were detected following their electrophoretic separation using a partial-denaturing gelatin-SDS-PAGE assay system [26], which consisted of $12 \%(\mathrm{w} / \mathrm{v})$ acrylamide mini-gels $(0.5 \mathrm{~mm})$ containing $0.2 \%(\mathrm{w} / \mathrm{v})$ gelatin, Tris- $\mathrm{HCl}$ buffer, $\mathrm{pH}$
8.8, and $0.1 \%(\mathrm{w} / \mathrm{v})$ SDS. Total protein from whole cell homogenates or magnetically isolated endosomes were solublilized, without heating, in Laemmli buffer [27] containing $0.35 \mathrm{M}$ (final concentration) 2-mercaptoethanol. Typically $10 \mu \mathrm{g}$ of total protein was electrophoresed per lane of the gels. In some instances, homogenates or purified endosomes were pre-incubated with the cysteine protease inhibitor, trans-epoxysuccinyl-L-leucylamido-(4-guanadino)butane (E-64; $10 \mu \mathrm{M})$, prior to solubilization in loading buffer. Following electrophoresis, gels incubated at $25^{\circ} \mathrm{C}$ in $2.5 \%(\mathrm{~V} / \mathrm{V})$ Triton X-100 for $30 \mathrm{~min}$, and then in $0.1 \mathrm{M}$ sodium acetate/acetic acid buffer containing $1 \mathrm{mM}$ dithiothreitol for $16 \mathrm{~h}$. To visualize proteolyzed bands the gels were stained with Coomassie Brilliant Blue $(0.25 \%(\mathrm{w} / \mathrm{v})$ in $50 \%(\mathrm{v} / \mathrm{v})$ methanol, $10 \%(\mathrm{v} / \mathrm{v})$ acetic acid) and destained with a solution of $50 \%$ $(\mathrm{v} / \mathrm{v})$ methanol and $10 \%(\mathrm{v} / \mathrm{v})$ acetic acid.

\subsection{Cloning and analysis of Rab cDNA clones}

Genomic DNA was isolated from E. histolytica trophozoites as described [28]. PCR was performed using genomic DNA as a template and degenerate sense and antisense primers corresponding to the amino acid sequences, GVGK(T/ S) and GNKCD, respectively. Both of these amino acid sequence motifs are highly conserved in Rab family proteins. The PCR products were sequenced as described [24]. An E. histolytica cDNA library (gift from Dr E. Tannich, Bernhard Nocht Institute for Tropical Medicine, Hamburg, Germany), prepared from amoebic trophozoites was screened as described [24] with ${ }^{32} \mathrm{P}$ end-labeled oligonucleotides (5' -TTAAGATTTACA CGAAATGAATTTGATGCA-3', 5' GATAGACAAGAGAGTTTTGA-3') that were designed using sequence information obtained from the PCR products described above. Positive clones were isolated and sequenced on both DNA strands as described [24].

Searches of the National Center for Biotechnology Information data bases were conducted online using BLAST ${ }^{\circledR}$ version 2.0. Alignments of amino acid sequences were generated using Mac Vector ${ }^{\circledR}$ (version 4.1.1) using the method of Wilbur and Lipman 
[29]. Phylogenetic analysis was carried out using PCGENE®, V6.85 (Intelligenetics. Inc.).

\subsection{SDS-PAGE and Western blot analysis}

Magnetically isolated endosomes were solubilized in Laemmli buffer [27] and SDS-PAGE was performed using the conventional discontinuous buffer system described by Laemmli [27]. Typically, $50 \mu \mathrm{g}$ of total protein was electrophoresed per lane in $12 \%$ (w/v) polyacrylamide gels. Proteins, resolved by SDS-PAGE were electroblotted onto nitrocellulose membranes in a Towbin buffer system [30] at $100 \mathrm{~V}$, for $1 \mathrm{~h}$, at a controlled temperature of $15^{\circ} \mathrm{C}$. Nitrocellulose-immobilized proteins were used in Western analysis.

The generation of polyclonal antibodies recognizing the $27-\mathrm{kDa}$ cysteine protease is described elsewhere [12]. Polyclonal antibodies recognizing EhRab11 or EhRab7 were generated by immunizing New Zealand male rabbits (Cocalico Biologicals, Reamstown, PA) with bacterially expressed (recombinant) antigen corresponding to amino acids 145206 in the C-terminal divergent region of EhRab11 or a synthetic peptide corresponding to amino acids 121-141 in a divergent region of EhRab7 [19]. For Western blot analysis, nitrocellulose membranes containing electroblotted proteins were blocked overnight in TBSTG buffer (10 mM Tris base, 150 $\mathrm{mM} \mathrm{NaCl}, 0.05 \%$ (v/v) Tween-20, 0.1\% (w/v) gelatin) at $4^{\circ} \mathrm{C}$. After blocking, the blots were incubated in primary antibody (either a 1:100 dilution in TBSTG of the polyclonal antiserum recognizing the EhRabs or a 1:1,000 dilution in TBSTG of the polyclonal antiserum recognizing the $27-\mathrm{kDa}$ cysteine proteinase), washed and then incubated in TBSTG supplemented with goat anti-rabbit antibodies (1:30,000 dilution in TBSTG, Sigma, St. Louis, MO). Blots were developed in NBT buffer (100 mM Tris base, $100 \mathrm{mM} \mathrm{NaCl}, 5 \mathrm{mM} \mathrm{MgCl}{ }_{2}$ containing the alkaline-phosphatase substrates BCIP (1.2 mM, Amresco, Solon, $\mathrm{OH})$ and nitroblue tetrazolium (0.6 $\mathrm{mM}$, Amresco, Solon, OH). Scanning densitometry and analysis was carried out using an AlphaImager ${ }^{\circledR} 2000$ (Alpha Innotech, San Leandro, CA). Standard curves obtained by scanning densitome- try of Western blots using known amounts of protein were linear. All densitometric values obtained by scanning Western blots of the 27-kDa cysteine protease, EhRab11 or EhRab7 were within this linear range (data not shown).

\subsection{Statistical analysis}

Student's $t$-test performed using the computer program GraphPAD Instat ${ }^{\circledR}$ (Version 1.12a, IBM®).

\section{Results and discussion}

\subsection{Magnetic fractionation of endosomal compart- ments of E. histolytica}

Magnetic separation of pinocytic vesicles of defined age from $E$. histolytica has previously been reported [31]; however, in this previous study the recovery of vesicles was approximately $25 \%$ and limited characterization of the purified vesicles was carried out. We sought to isolate and extensively characterize early and late endosomal vesicles from E. histolytica using a magnetic fractionation protocol that we have previously employed to isolate pure endosomes from a non-pathogenic amoeba Dictyostelium discoideum with high yield ( $>75 \%$ ) [24].

To determine if colloidal iron-dextran was toxic to E. histolytica trophozoites, we determined the generation time of the amoebae in the presence of the magnetic probe. E. histolytica trophozoites were grown in TYI-S-33 nutrient medium with or without iron-oxide-dextran $(2 \mathrm{mg} / \mathrm{ml})$. At various time points over $72 \mathrm{~h}$, the cells were harvested and the average number of cells present in the culture was estimated by cell counting. The doubling time for trophozoites in the absence and presence of the iron probe was calculated to be 12.8 and $11.8 \mathrm{~h}$, respectively, indicating that the magnetic probe did not significantly affect cell growth (data not shown). Similarly, the iron probe had no affect on the morphology of cells, as evidenced by microscopic examination of the cells at each time point during growth monitoring (data not shown). 

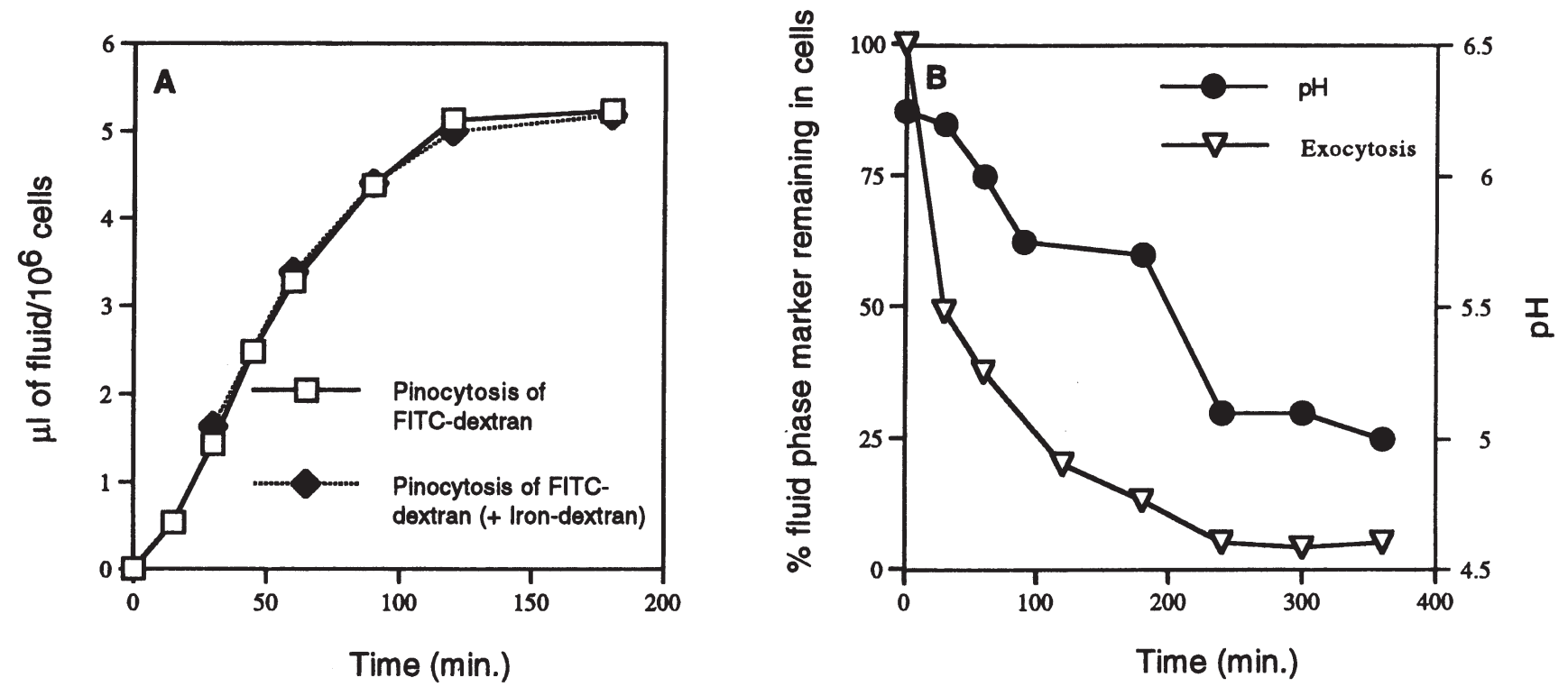

Figure 1. Characterization of the pinocytosis by E. histolytica. A: Pinocytosis of fluid phase was measured in the absence ( $\square$ ) or presence $(\$)$ of iron-dextran using FITC-dextran as a fluid phase marker. Colloidal iron-dextran had no adverse effects on pinocytic characteristics including rate of FITC-dextran uptake, half-time of saturation with FITC-dextran, and maximum saturation volume. B: Cells were pulsed for 3 days with FITC-dextran, and then chased for $6 \mathrm{~h}$ in fresh marker-free media. At the time points indicated during the chase period, the $\mathrm{pH}$ of the compartment harboring the marker $(\bullet)$ and the amount of marker remaining in the cells $(\mathrm{V})$ was measured. Average $\mathrm{pH}$ declined during the chase period reaching minimum value of 5.10 after $3 \mathrm{~h}$. Marker was rapidly exocytosed from the cell such that little remained after $4 \mathrm{~h}$ of chase.

Previously, it was reported that early endosomal compartments of E. histolytica rapidly exchange their fluid phase contents with the external milieu [6]. Steady state, signifying equal rates of uptake and egestion of fluid phase, is an indication of complete filling of this compartment and was reached in 2-3 $\mathrm{h}$ [6]. To determine if colloidal iron-dextran altered the pinocytic loading of early compartments, trophozoites were incubated with FITC-dextran with or without the magnetic probe. At the time points indicated (Figure 1A) samples of cells were taken and the level of intracellular fluorescence or iron was determined. The kinetics of ingestion of the FITC-dextran alone (Figure 1A, open squares) was similar to that reported by Aley et al. [6]. Uptake was linear for the first 60 min after which the rate of uptake slowed, reaching equilibrium at $2 \mathrm{~h}$ (Figure 1A). Steady state was reached at the equivalent volume of $5.2 \mu \mathrm{l}$ of fluid $/ 10^{6}$ cells and the halftime of filling with marker was approximately 50 min. Pinocytosis of FITC-dextran was not altered in the presence of colloidal iron as the rate of fluid phase uptake, half-time of saturation, and maximum saturation volume remained unchanged (Figure 1A, closed diamonds). These data indicate that the magnetic probe did not adversely affect pinocytosis by E. histolytica. The initial rate of uptake of the iron probe was identical to that of FITC-dextran indicating that the magnetic probe was not preferentially internalized as could have occurred had it become associated with cell surface proteins (data not shown).

It has been reported that the late acidic endosomal compartment of E. histolytica is a "slowloading/slow-release" compartment, and labeling of these vesicles with markers requires very long pulse times (days) [6]. To determine the optimal pulse/chase regime for labeling of late vesicles, cells were pulsed for 3 days in TYI-S-33 supplemented with FITC-dextran, and then chased for $6 \mathrm{~h}$ in fresh marker-free media. Samples were taken at hourly intervals, and the $\mathrm{pH}$ of the endosomal compartments and the amount of marker remaining in the cell was determined. At the begin- 
ning of the chase period the average $\mathrm{pH}$ of labeled compartments was determined to be 6.25 (Figure $1 \mathrm{~B}$, closed circles). As the marker was chased from the near-neutral early compartments, the average $\mathrm{pH}$ declined reaching a minimum value of 5.10 after $3 \mathrm{~h}$ (Figure 1B, closed circles). Thus, at $3 \mathrm{~h}$, late compartments were exclusively labeled. The $\mathrm{pH}$ remained low for up to $6 \mathrm{~h}$ of chase, however, very little marker remained in the cell after $4 \mathrm{~h}$ (Figure $1 \mathrm{~B}$, open triangles). Thus, optimal loading of late compartments of E. histolytica occurred after a 3-day pulse with marker and a 3-h chase.

Trophozoites were incubated for $2 \mathrm{~h}$ with ironoxide-dextran to load early compartments or were incubated for 3 days with the probe and chased for $3 \mathrm{~h}$ in fresh marker free medium for $3 \mathrm{~h}$ to label late compartments. Fed cells were immediately washed in ice cold buffer $\mathrm{H}$ and homogenized by passage through a polycarbonate filter. It was determined that $90 \%$ of the iron probe was pelletable from the homogenate by centrifugation (data not shown); therefore, organelle disruption prior to magnetic fractionation was minimal. Iron-containing endosomes were then isolated by magnetic fractionation as previously described $[20,24]$. Recovery of endosomal vesicles (estimated by measuring recovery of elemental iron) was approximately $57 \%$ for early vesicles and $57 \%$ for late vesicles. It is postulated that this improved yield over the previously reported method [31] is due to the higher osmolarity of the homogenization buffer utilized in this study which afforded better protection to the iron containing endosomes during the procedure. It was determined that incomplete recovery observed in this study was due to breakage of the vesicles during magnetic fractionation and not due to a lack of column binding by iron-containing vesicles as iron in the flow-through could not be pelleted by centrifugation. Cross-contamination between the two compartments (estimated by differential loading of the two compartments with FITC- and iron-dextrans) was negligible as was contamination with plasma membrane (estimated by labeling the cell surface $\left(\right.$ at $\left.4^{\circ} \mathrm{C}\right)$ with FITC-succinyl-concanavalin A after iron uptake; data not shown).

\subsection{Enrichment of hydrolases in early and late endosomes}

Since endocytic compartments are typically characterized by their enrichment of acid hydrolases, we first measured acid phosphatase in purified early and late endosomes. We found that acid phosphatase activity was enriched in both early and late endosomes as compared to whole cell homogenates (Figure 2). The enrichments were 2- and 2.5-fold for early and late endosomes, respectively, and statistical analysis indicated that these enrichments were significant (Figure 2). Anaya-Ruiz et al. [14] recently demonstrated that $83.7 \%$ of total $E$. histolytica acid phosphatase was associated with intracellular membranes. Furthermore, it was shown that this enzyme had optimum activity at an acidic $\mathrm{pH}$ of 5.5. Taken together, these data suggest that acid phosphatase of E. histolytica may reside in endo-lysosomal-like compartments. The results of this study provide further evidence that acid phosphatase may be sequestered in such compartments as evidenced by its enrichment in magnetically purified endosomes.

The recovery of total cellular acid phosphatase in purified early and late endosomal vesicles was $17 \%$ and $15.5 \%$, respectively. The recovery of acid phosphatase activity in the flow-through fraction (non-endosomal components and lysed endosomes) was approximately $70 \%$ (data not shown). The acid phosphatase-containing intracellular membrane fraction, described by Anaya-Ruiz et al. [14], would include the membranes of early endosomal vesicles, late endosomal vesicles and vesicles of other membrane trafficking systems such as biosynthetic or secretory pathways that may or may not communicate with the endo-lysosomal system. Since only $83.7 \%$ of cellular acid phosphatase activity associates with inner membranes [14] and since the recovery of endosomes in this study was approximately $57 \%$ (Section 3.1), recovery of acid phosphatase in purified endosomal fractions could not be expected to exceed $47.7 \%$ of total cellular activity. Moreover, recovery of acid phosphatase activity in purified early and late endosomes would represent only that portion of the enzyme that is associated with the compart- 


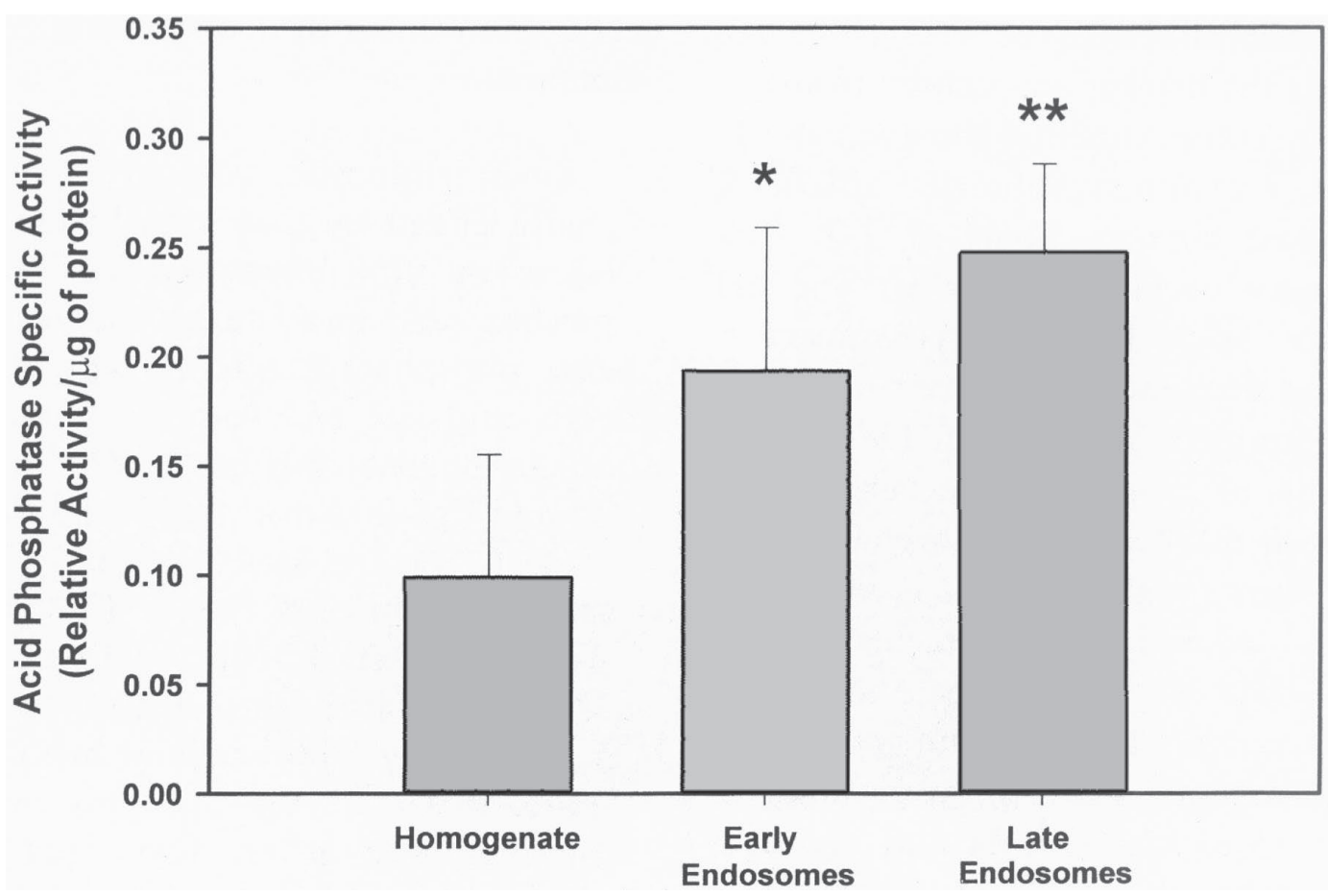

Figure 2. Enrichment of acid phosphatase activity in magnetically purified endosomes of E. histolytica. Acid phosphatase activity was measured in whole cell homogenate and magnetically purified early and late endosomes. Each bar represents the mean \pm S.D. of four experiments. Acid phosphatase activity was significantly enriched in early endosomes $\left({ }^{*} P<0.05\right)$ and very significantly enriched in late endosomes $\left({ }^{* *} P<0.01\right)$ as compared to homogenate.

ment (early or late) of interest. The similar recovery of acid phosphatase in early and late vesicles suggests that equivalent levels of acid phosphatase are associated with early and late endosomes.

Acid and neutral proteases ranging in molecular weight from 16 to $116 \mathrm{kDa}$ have been identified in extracts of E. histolytica [3]. Since endocytic compartments are typically characterized by their acidity, we measured acid protease activity in purified endosomes using partial-denaturing gelatin SDSPAGE. Protease activity in the range of $29-68 \mathrm{kDa}$ was found to be enriched in early endosomes as compared to whole cell homogenates (Figure 3A). These activities were inhibited by pre-incubation of the purified endosomes with a potent specific inhibitor of cysteine proteases, E-64, suggesting that gelatin hydrolysis came about by the action of one or more cysteine proteases (Figure 3B). This group of proteases was also observed in the flow through fraction (non-endosomal components and lysed endosomes) but was not enriched as compared to whole cell homogenates (data not shown).
A doublet of gelatinoytic activity at 44 and 46 $\mathrm{kDa}$ demonstrated the greatest enrichment among the observed proteases. The gelatinolytic activity observed in the 29-35-kDa range is consistent with the mobility and activity of the $27-\mathrm{kDa}$ cysteine protease in non-denaturing or partial-denaturing gelatin SDS-PAGE systems [11] suggesting that this important virulence factor is enriched in this endocytic compartment of E. histolytica. Higher molecular weight (> $35 \mathrm{kDa}$ ) cysteine protease activities that were observed to be enriched in early endosomes are consistent with the results of Li et al. [11], Ankri et al. [32], and de Meester et al. [33]. All but three of these cysteine protease activities, the 68-, 46- and 29-kDa species (Figure 3A, solid arrows), were also enriched in late endosomes of E. histolytica. The enrichment of proteases in early endosomes and not in late endosomes is not unprecedented; macrophages demonstrate differential enrichment of cathepsins in early and late endosomes and lysosomes [34]. In both E. histolytica and macrophages, the physiological relevance of this differential enrichment is not 


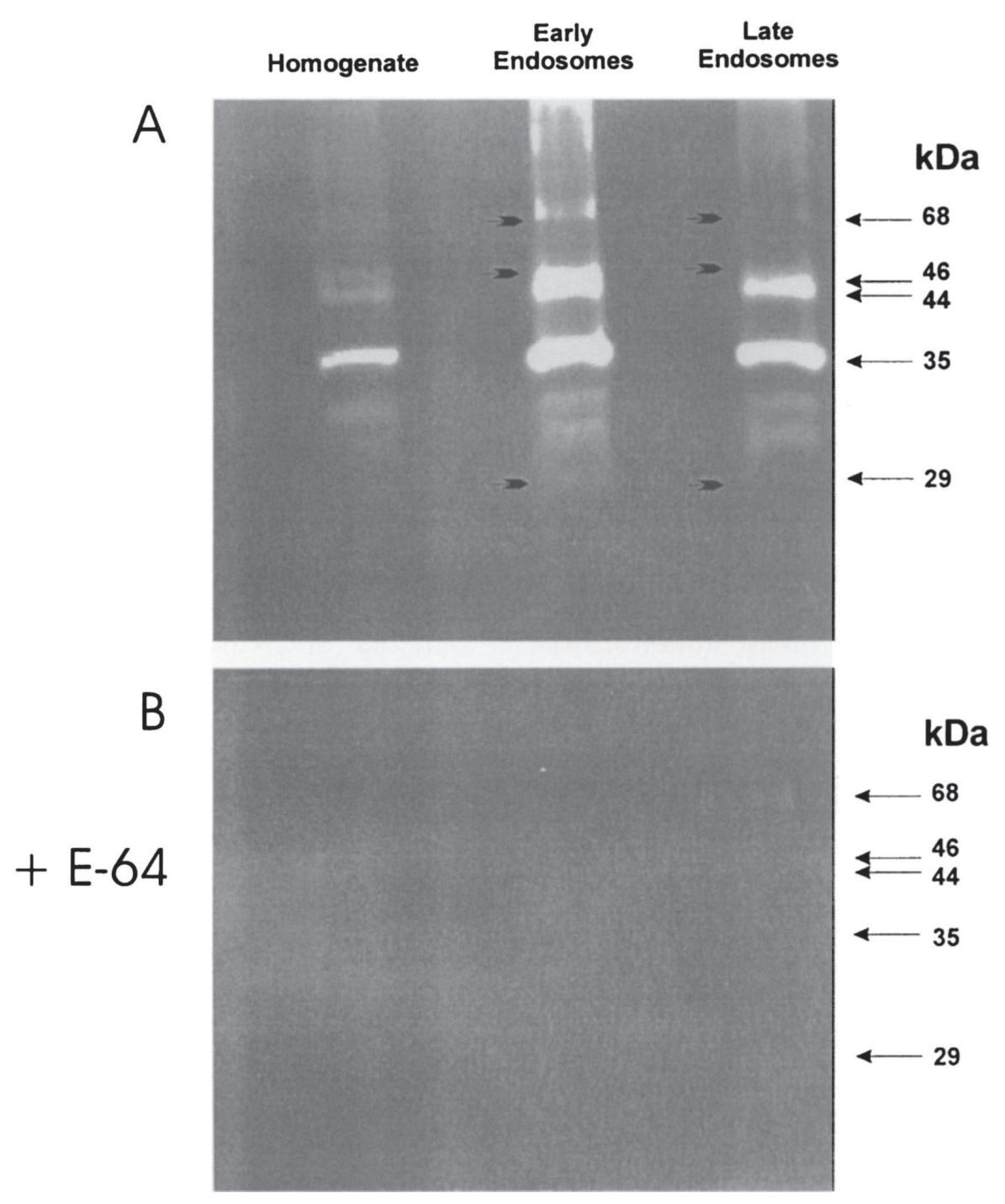

Figure 3. Gelatin SDS-PAGE demonstrating acid cysteine protease activity in magnetically purified endosomes of E. histolytica. Total protein $(10 \mu \mathrm{g}$ /lane) from whole cell homogenate and magnetically purified early and late endosomes (A) or total protein from these fractions pre-incubated with a cysteine protease inhibitor, E-64 (B), were resolved under partial-reducing conditions on polyacrylamide gels containing $0.2 \%(\mathrm{w} / \mathrm{v})$ gelatin as described [26]. The apparent molecular mass (in $\mathrm{kDa})$ is shown on the right. A number of cysteine proteases in the $29-68-\mathrm{kDa}$ range were found to be enriched in early endosomes as compared to whole cell homogenates (A) and inhibited by pre-incubation with E-64 (B). All but three of these cysteine proteases, the 68-, 46and 29-kDa species (A, solid arrows), were also enriched in late endosomes of E. histolytica.

known and the mechanisms by which proteases are retained in various endosomal compartments remains to be studied.

Previously, it had been shown by immuno-electron microscopy that a major $30 \mathrm{kDa}$ cysteine protease was localized to clear cytoplasmic zones not limited by membrane, but translocated to phagosomes after trophozoites ingested erythrocytes [35]. Interestingly, cysteine protease activity in the 30-
$\mathrm{kDa}$ range was found to be enriched in the magnetically purified vesicles (Figure 3A) suggesting that this activity may also translocate to vesicles of pinocytic origin and that during maturation pinosomes may be modified in a manner similar to that for phagosomes.

de Meester et al. [33] have reported that several additional cysteine proteases (56, 40, and $39 \mathrm{kDa}$ ) are associated with E. histolytica. This was demon- 


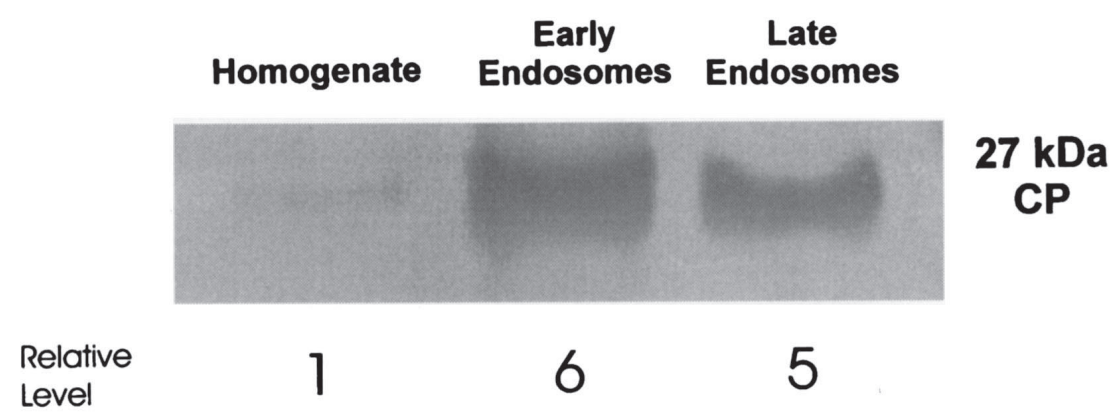

Figure 4. Western blot analysis of a lysate of E. histolytica trophozoites (homogenate) and magnetically purified early and late endosomes to determine the enrichment of the $27-\mathrm{kDa}$ cysteine protease (cp). Proteins from these fractions were resolved by SDSPAGE, transferred to nitrocellulose membranes and decorated with a polyclonal antibody recognizing the 27-kDa cysteine protease [12]. The relative level of the antigen (indicated below the blot) was determined by scanning densitometry and the level of protease in the homogenate was given an arbitrary value of 1 . The $27-\mathrm{kDa}$ cysteine protease was enriched in early and late endosomes as compared to whole cell homogenate.

strated by incubating cells with an ${ }^{125}$ I-labeled, irreversible, cysteine protease inhibitor, followed by SDS-PAGE of trophozoite extracts and autoradiography. In the present study we did not observe these cysteine proteases. This difference may be due to the fact that the radiolabeling protocol described by de Meester et al. [33] would not distinguish between neutral and acid cysteine proteases, while the gelatin SDS-PAGE employed in this study would only reveal acid cysteine protease activity. Therefore, these four additional proteases may have neutral $\mathrm{pH}$ optima and would not be observed under the conditions of gelatin hydrolysis employed in this study. Alternatively, since partial-reducing conditions were used in this study to resolve protease activity and de Meester et al. [33] employed reducing conditions, the absence of the 55-, 40-, and 39$\mathrm{kDa}$ proteases observed in this work may be due to differences in mobility of these activities in different gel systems.

To confirm the enrichment of the 27-kDa cysteine protease in early and late endosomes of E. histolytica, Western blot analysis of purified vesicles was carried out using anti-27 kDa cysteine protease antibodies [12]. Scanning densitometry of the Western blot indicated that this protease was enriched six-fold in early vesicles and five-fold in late vesicles (Figure 4). These data correlate well with the enrichment of protease activity in the 29-35-kDa range on gelatin SDS-PAGE gels (Figure 3).

In many eukaryotic cells, secretory and endosomal pathways may merge. Newly synthesized precursors of hydrolases are phosphorylated and sulfated on oligosaccharide side-chains in the Golgi, proteolytically cleaved in endosomal compartments, and then transported to dense secondary lysosomes where processing is completed. Acid phosphatase [13] and the 27-kDa cysteine protease [12] are secreted proteins and were found to be enriched in purified endosomal vesicles suggesting that secretory and endocytic pathways may also merge in $E$. histolytica.

\subsection{Cloning and sequencing of Rab cDNA clones}

Rab GTPases play important roles in endosomal vesicle trafficking. It is likely that Rab-like GTPases regulate similar processes in E. histolytica; however, to date only one Rab, a Rab7-related GTPase, has been identified in this organism [19], and its function has not been discerned. To begin to identify the role of Rab GTPases in endosomal trafficking of E. histolytica, we sought to identify additional Rab GTPases.

Portions of two Rab genes from E. histolytica (EhRabs), 321 and 327 base pairs long, were isolated from a set of PCR products amplified from E. histolytica genomic DNA (data not shown). PCR was performed using degenerate sense and antisense primers corresponding to the amino acid sequences, GVGK(T/S) and GNKCD, respectively. Both of these amino acid sequence motifs are highly conserved in Rab family proteins. The PCR products were sequenced and the ammo acid 
sequences predicted to be encoded by these PCR products demonstrated a high degree of homology with various heterologous Rab proteins (data not shown). An E. histolytica cDNA library was screened with ${ }^{32} \mathrm{P}$ end-labeled oligonucleotides that were designed using sequence information obtained from the PCR products described above. From an initial screen of 30,000 clones, two positive clones, termed EhRab11 and EhRabA, were isolated and sequenced on both DNA strands (Figure 5). The original oligonucleotide sequences were found within the nucleotide sequences of the two clones, thus supporting the authenticity of the clones (Figure 5, underlined sequences). The precise nucleotide sequence of the PCR-generated fragments was found in the nucleotide sequence of the corresponding cDNAs clones.

\subsection{Deduced EhRab11 polypeptide and homology}

The EhRab11 clone (660 base pairs; GenBank Accession No. AF03018) contained a single open reading frame predicted to encode a polypeptide of at least 206 amino acids with a molecular mass of at least $23.2 \mathrm{kDa}$ as the start codon was missing from the cDNA clone (Figure 5). Using the same cDNA library, Lohia and Samuelson [36] have isolated full length and 5 ' truncated clones encoding several other small molecular weight GTPases including, Eh rac $A$, Eh racB, Eh racC, and Eh racD. Attempts to recover a full-length EhRab11 clone are currently underway.

Phylogenetic analysis was performed to identify Rab proteins to which EhRab11 was most similar. Such information may provide insight into the evolutionary origin of the EhRab11 gene or the function of its associated gene product. EhRab11 clustered with a group of Rab 11-related proteins and was closely related to the Rab 11 homologs of Saccharomyces cerevisiae and Plasmodium falciparum (Figure 6). These Rab11-related proteins and EhRab11 share at least $52 \%$ identity and $70 \%$ similarity at the amino acid level (Figure 7). The relatedness of EhRab11 to other Rab 11 proteins was further confirmed by aligning the predicted amino acid sequence of EhRab11 with Rab 11 proteins from various species (Figure 7). The alignment revealed that EhRab11 was highly related along the entire length of the deduced amino acid sequence to these Rab 11-like proteins. By comparison with other Rab proteins, it is predicted that codons for three to six amino acids are missing from the $5^{\prime}$ end of the EhRab11 clone. Therefore the predicted molecular weight of the full-length gene produce of EhRab11 is between 26.5 and $29.8 \mathrm{kDa}$.

\subsection{Conserved domains of EhRab11}

The alignment also revealed that EhRab11 displays many of the structural features characteristic of small molecular weight GTP-binding proteins (Figures 5 \& 7) and in particular, Rab GTPases. Firstly, the four domains which were originally identified in the small molecular weight GTPase, p21 Ras, [37] and are proposed to participate in the binding of GTP/GDP, are highly conserved in EhRab11 (Figures 5 \& 7). These domains include the highly conserved WD-TAGQE (region II) amino acid sequence motif which is proposed to interact with the $\gamma$-phosphate of GTP, the NKXD (region III) sequence, also termed the guanine specificity region, and the EXSAK/L (region IV) sequence which is proposed to interact with the guanine base. While the guanine binding loop, characterized by the amino acid sequence GXXXXGKS/T (region I), is the most variable of the four conserved domains, this motif is also highly conserved in EhRab11.

In addition to the four GTP binding domains, EhRab11 also contains a highly conserved arginine residue and phenylalanine residue, following the second and fourth GTP binding domains, respectively (see asterisks $\left({ }^{*}\right)$, Figure 7). EhRab11 also contains a carboxy terminal "-cysteine-cysteine" motif (Figures 5 \& 7) which is characteristic of Rab GTPases and is essential for proper membrane localization and normal functioning of the protein.

The putative effector domain of small molecular weight GTPases, which is the site of interaction with effector proteins and possibly regulatory proteins such as activators or inhibitors, is a less conserved region among members of the Rab family of GTPbinding proteins. This region is thought, however, to provide the signature sequence differentiating the 


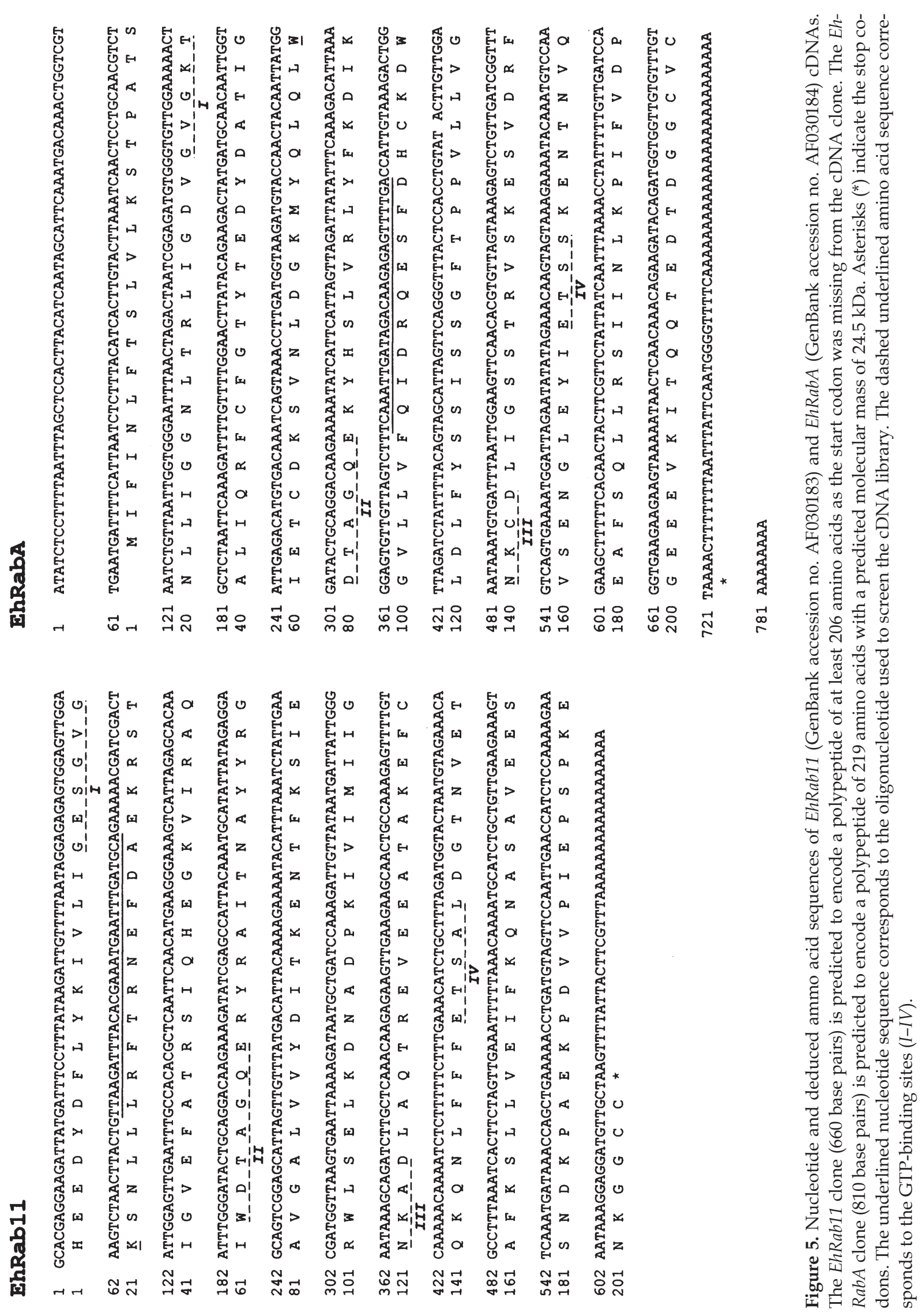




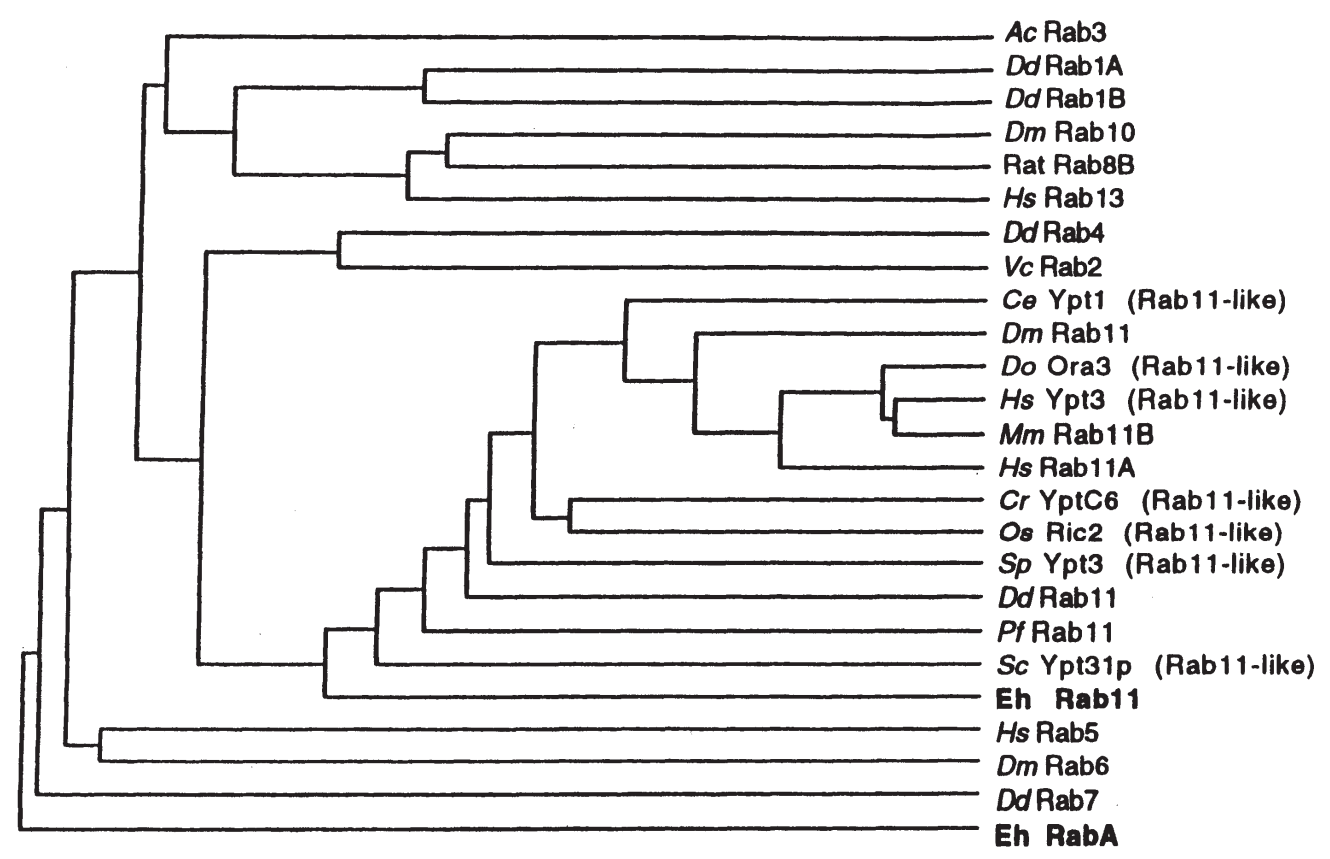

Figure 6. Phylogenetic analysis of EhRab11 and EhRabA predicted amino acid sequences. GenBank Accession numbers are given in square brackets ([ ]). A progressive alignment of Rab-like proteins from E. histolytica [Eh Rab11 = AF30183; Eh RabA = AF30184], a marine gastropod [Aplysia californica (Ac), Rab3 = U00986], Dictyostelium discoideum (Dd) [Rab1A = L21009; Rab1B = L21010; Rab4=U02927; Rab11 = U02925; Rab7 = U02928], Drosophila melanogaster $($ Dm $)$ [Rab10 = 2317272; Rab11 =D84315; Rab6 = 2317272], Rat [Rab8b = 1314638], Homo sapiens (Hs) [Rab13 = 1710016; Ypt3 = X79780; Rab11A = AF000231; Rab5 = 642532], a green alga [Volvox carteri $(V c)$ ] Rab2 = 549811], Caenorhabditis elegans (Ce) [Ypt1 = AF003139], electric ray [Discopyge ommata (Do), Ora3 = M38392], Mus musculus (Mm) [Rab11B = L26528], a green alga [Chlamydomonas reinhardtii (Cr), YptC6 = U13169], rice [Oryza saliva (Os), Ric2 = D13858], Schizosaccharomyces pombe (Sp) [Ypt3 = X52100], Plasmodium falciparum (Pf) [Rab11 = X93161], Saccharomyces cerevisiae (Sc) [Ypt31p = U18778, U00092]. The progressive alignment was drawn using PCGENE®, V6.85 (Intelligenetics, Inc.).

various classes of Rab proteins. The putative effector domain of EhRab11 is identical to those of other Rab11-related proteins (Figure 7) further confirming the relatedness of the Entamoeba protein and other Rab11 species. Together these data suggest that EhRab11 is an authentic Ras-related GTP-binding protein of the Rab family and that EhRab11 is a Rab11 homolog for Entamoeba.

\subsection{Deduced EhRabA polypeptide and homology}

The EhRabA clone (810 base pairs, GenBank Accession No. AF030184) contained a single open reading frame predicted to encode a polypeptide of 219 amino acids with a predicted molecular mass of 24.5 $\mathrm{kDa}$ (Figure 5). The predicted translation product of EhRabA, EhRabA, contains many of the hallmarks of Rab GTPases, including a carboxy terminal -cyste-
ine-X-cysteine motif and the four GTP-binding domains (Figure 5).

A search of the NCBI database revealed that EhRabA shares less than $47 \%$ amino acid sequence identity with other existing Rab proteins (data not shown). Furthermore, phylogenetic analysis demonstrated that EhRabA showed only limited relatedness to other select Rab proteins (Figure 6). Taken together these results suggest that EhRabA is a novel member of the Rab family of GTP-binding proteins.

\subsection{Enrichment of Entamoeba Rab GTPases in puri- fied endosomal compartments}

In mammalian cells, Rab 11 has been localized to constitutive and regulated secretory vesicles [38] but its exact function in secretion has not been dis- 
ఏ

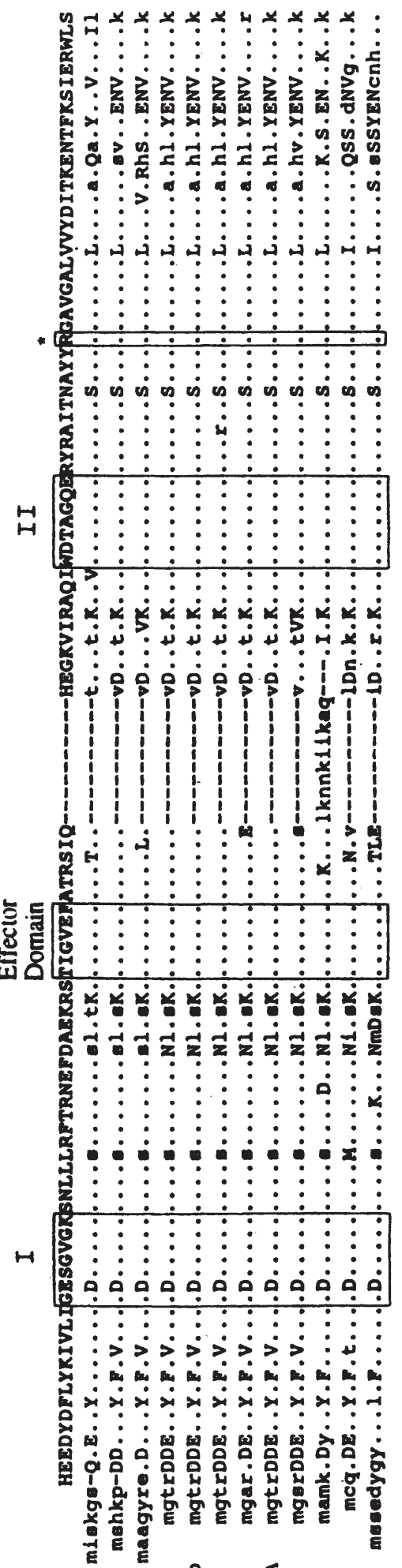

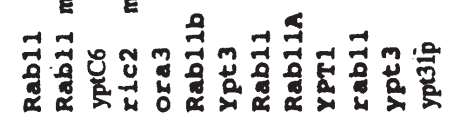

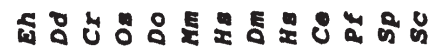

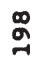

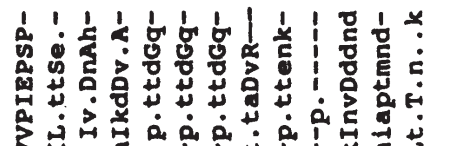

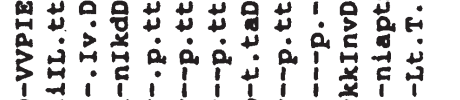

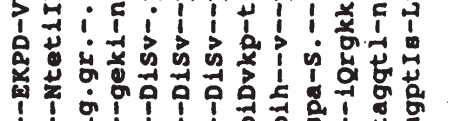

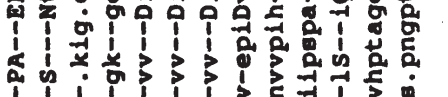

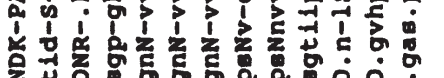

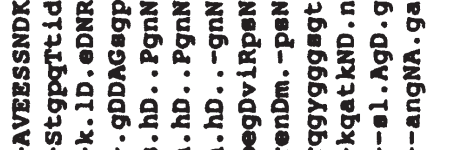

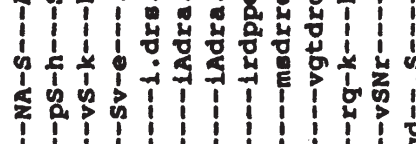

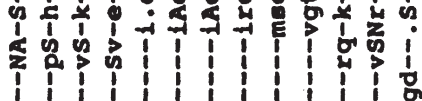

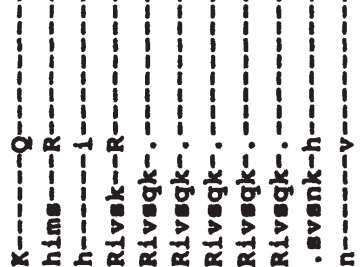

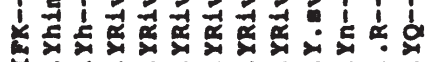

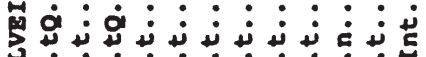

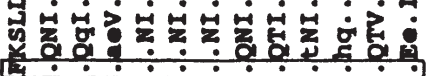

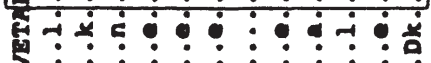

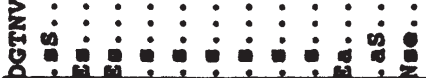

只展

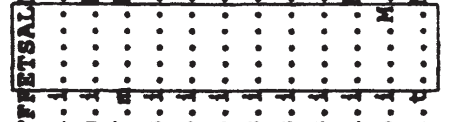

+

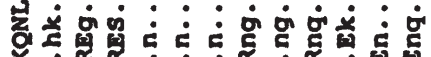

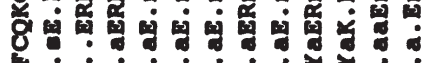

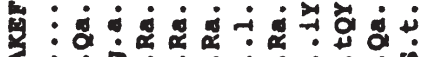

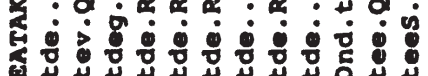

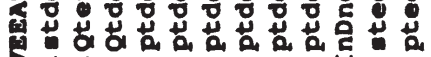

过

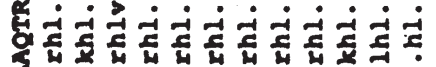

品

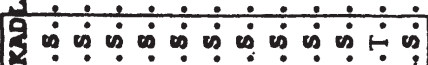

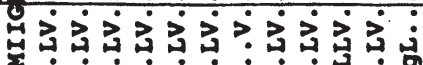

$3 \vdots \vdots \vdots \vdots \vdots \vdots \vdots \vdots \vdots \vdots \vdots \vdots 0$

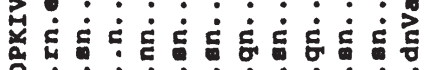

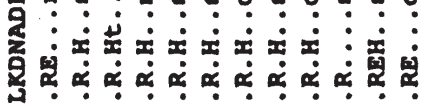

苗:: : : : : : ::: : :

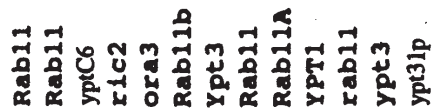

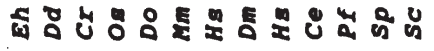

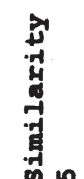

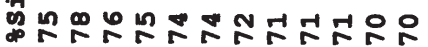

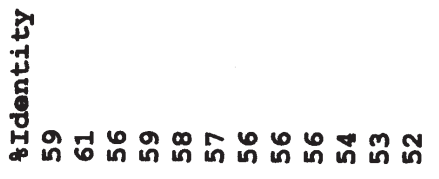

ัั

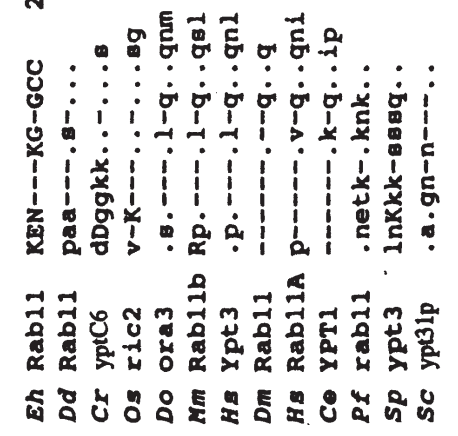

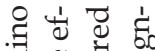

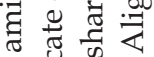

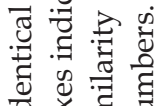

- ํํำ

ฮु

to

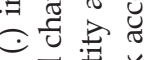

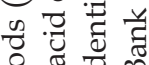

हैं 유

ळं छ్

$\triangle \overrightarrow{0}$

के त्र

政

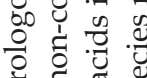

बै

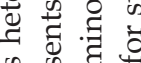

जे के चै

륨 ठ

bo

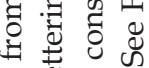

密

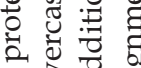

$\exists$ है

त्)

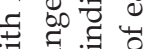

3 등 형

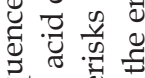

웜

कृ छ

न $\overrightarrow{0} \cdot \overrightarrow{0}$

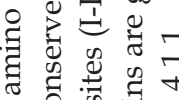

पु 0 is 0

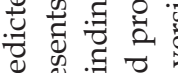

若范

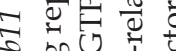

है

进哠

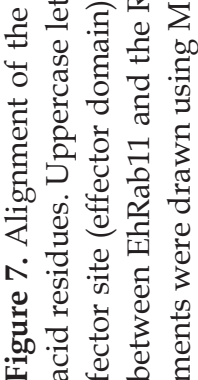




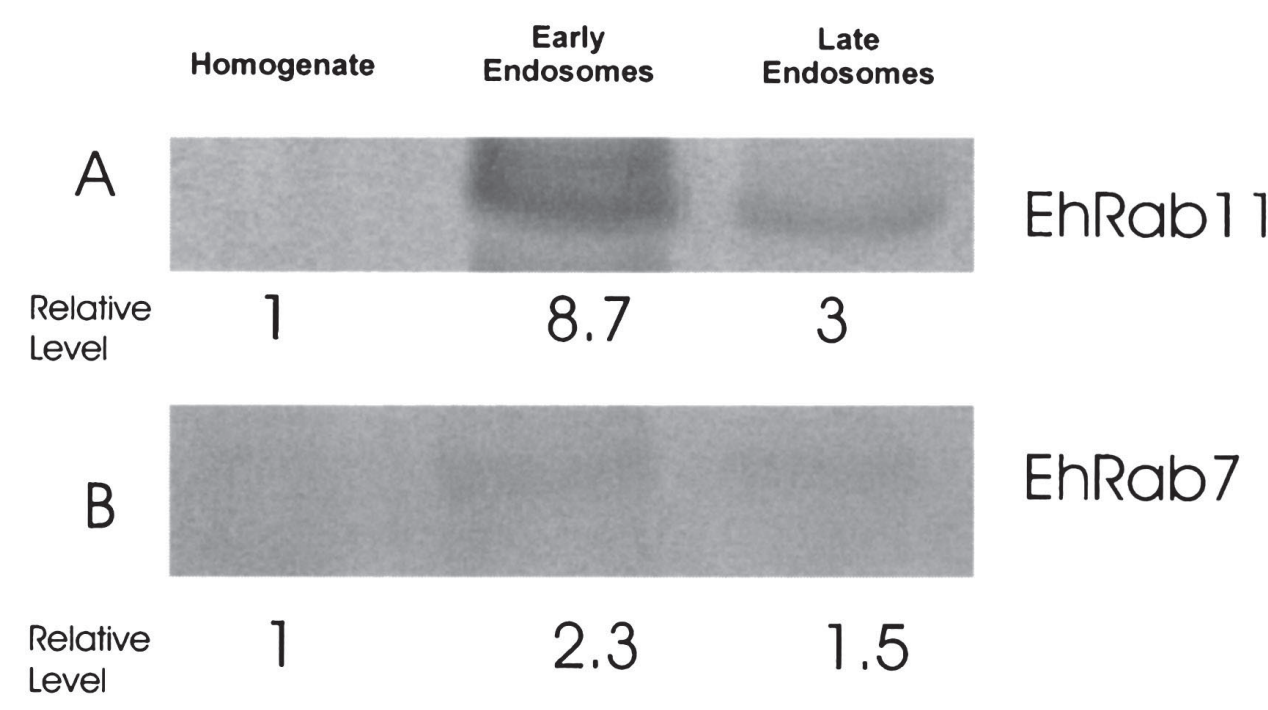

Figure 8. Western blot analysis of a lysate of E. histolytica trophozoites (homogenate) and magnetically purified early and late endosomes to determine the enrichment of the Rab11-like and Rab7-like GTPases. Proteins from these fractions were resolved by SDS-PAGE, transferred to nitrocellulose membranes and decorated with a polyclonal antibody recognizing EhRab11 or EhRab7. The antibodies generated to EhRab11 and EhRab7 recognized single bands of 28.5 and $24 \mathrm{kDa}$, respectively. The relative level of the antigen (indicated below the blot) was determined by scanning densitometry and the levels of the Rab GTPases in the homogenate were given an arbitrary value of 1 . EhRab11 and EhRab7 were enriched in early and late endosomes as compared to whole cell homogenate.

cerned. More recently, Rab11 has been demonstrated to regulate membrane traffic through the recycling endosome in mammalian cells [39]. Rab7like GTPases have also been shown to play roles in early endosome function [40-42]. To determine if the E. histolytica Rab11-like and Rab7-like GTPases localized to endosomal compartments, we generated antibodies that were highly specific for the Cterminal divergent regions of EhRab11 and EhRab7 and performed Western blot analysis of magnetically purified endosomes. The antibodies generated to EhRab11 and EhRab7 recognized single bands of 28.5 and $24 \mathrm{kDa}$, respectively. Consistent with their proposed function in mammalian cells, EhRab11 was enriched 8.7- and 3-fold in purified early and late endosomes of E. histolytica, respectively (Figure 8). EhRab7 was enriched 2.3- and 1.5-fold in early and late endosomes, respectively (Figure 8). These data suggest that these Rabs may play a role in vesicle trafficking between early and late compartments of E. histolytica. This is the first subcellular localization of any Rab GTPase of this organism. EhRab11 and EhRab7 were also detected in the flow through fraction (non-endosomal components and lysed en- dosomes) but were not enriched as compared to whole cell homogenates (data not shown).

\section{Conclusions}

We have isolated and characterized early and late pinosomal compartments of E. histolytica and demonstrated that they were enriched in acid phosphatase activity and a number of cysteine proteases including the 27-kDa cysteine protease, proposed to be important in pathogenicity. In many eukaryotes, intermediate and mature forms of hydrolases reside in organelles that are part of or communicate with the endosomal pathway. The data presented in this study suggest that E. histolytica hydrolases may also reside in a compartment that is part of the endosomal pathway.

We also described the cloning and characterization of cDNAs encoding a Rab11-like member and a novel member (EhRabA) of the Ras-related Rab family of GTPases from E. histolytica. Western blot analysis demonstrated that the Rab11-like and a previously described Rab7-like [19] GTPases were 
enriched in early and late compartments. These data suggest that these Rabs may function in pinocytosis and/or trafficking between the early and late compartments of E. histolytica. Although Western blot analysis indicated that EhRab11 and EhRab7 associate with the endo-lysosomal system of E. histolytica, it is conceivable that EhRab11 and/ or EhRab7 may also be localized to and function in vesicular movement of other membrane systems that may communicate with the endo-lysosomal system by vesicle trafficking. Analysis of the potential subcellular localization of these Rabs in the endoplasmic reticulum, Golgi, or secretory membrane systems is currently underway. The physiological relevance of the novel Rab, EhRabA, is not known however, subcellular localization of this protein may provide insight into its function. The identification of this novel Rab is a potentially important finding as proteins exclusive to E. histolytica may serve as targets for the future development of anti-amoebic protocols.

Mutational analysis of the individual Rab proteins of E. histolytica will provide insight into protein and vesicle trafficking in this organism. These processes are critical in the pathogenicity of this organism and elucidation of the mechanisms governing membrane trafficking is crucial to understanding the biology of the parasite and the disease that it causes.

\section{Acknowledgments}

The cDNA library was obtained from Dr. E. Tannich, (Bernhard Nocht Institute for Tropical Medicine, Hamburg, Germany). This work was supported by grants from the NSF (DCB-9104576) and NIH (DK 39232-05) to J. A. Cardelli. The authors thank Dr. T. Tanaka (Division of Host Defence Mechanism, Tokai University School of Medicine, Kanagawa, Japan) for the EhRab7 clone [19]. The authors acknowledge the support Feist-Weiller Cancer Research Center of LSUMC and the Center for Excellence in Arthritis and Rheumatology of LSUMC.

\section{References}

[1] Walsh JA. Problems in recognition and diagnosis of amebiasis: estimation of the global magnitude of morbidity and mortality. Rev Infect Dis 1986; 8:228-38.

[2] Ravdin JI. Amebiasis. Clin Infect Dis 1995; 20:1453-66.

[3] Que X, Reed SL. The role of extracellular cysteine proteinases in pathogenesis of Entamoeba histolytica invasion. Parasitol Today 1997; 13:190-4.

[4] Mirelman D. Ameba-bacterium relationship in amebiasis. Microbial Rev 1987; 51:272-84.

[5] Rodriguez MA, Orozco E. Isolation and characterization of phagocytosis- and virulence-deficient mutants of Entamoeba histolytica. J Infect Dis 1986; 154:27-32.

[6] Aley SB, Cohn ZA, Scott WA. Endocytosis in Entamoeba histolytica. Evidence for a unique non-acidified compartment. J Exp Med 1984; 160:724-38.

[7] Lohden-Bendinger U, Bakker-Grunwald T. Evidence for a vacuolar-type proton ATPase in Entamoeba histolytica. Z Naturforsch 1990; 45C:229-32.

[8] Walsh JA. Human infection by Entamoeba histolytica. In: Ravdin JI, editor. Amebiasis. Wiley, New York, 1988:93-105.

[9] Leippe M. Amoebapores. Parasitol Today 1997; 13:178-83.

[10] Leippe M, Sievertsen HJ, Tannich E, Horstmann RD. Spontaneous release of cysteine proteases but not of poreforming peptide by viable Entamoeba histolytica. Parasitology 1995; 111:569-74.

[11] Li E, Yang W-G, Zhang T, Stanley SL Jr. Interaction of laminin with Entamoeba histolytica cysteine proteinases and its effect on amebic pathogenesis. Infect Immun 1995; 63:4150-3.

[12] Stanley SL Jr, Zhang T, Rubin D, Li E. Role of the Entamoeba histolytica cysteine proteinase in amebic liver abscess formation in severe combined immunodeficient mice. Infect Immun 1995; 63:1587-90.

[13] Aguirre-Garcia MM, Rosales-Encina JL, Talamas-Rohana P. Secreted Entamoeba histolytica acid phosphatase (SAP). Arch Med Res 1997; 28 Spec No:184-5.

[14] Anaya-Ruiz M, Rosales-Encina JL, Talamas-Rohana P. Membrane acid phosphatase (MAP) from Entamoeba histolytica. Arch Med Res 1997; 28 Spec No:182-3.

[15] Pfeffer S. Rab GTPases: Master regulators of membrane trafficking. Curr Biol 1994; 6:522-6.

[16] Schimmoller F, Simon I, Pfeffer SR. Rab GTPases, directors of vesicle docking. J Biol Chem 1998; 273:22161-4.

[17] Khosravi-Far R, Lutz RJ, Cox AD, Conroy L, Bourne JR, Sinesky M, Balch WE, Buss JE, Der CJ. Isoprenoid modification of rab proteins terminating in CC or CXC motifs. Proc Natl Acad Sci 1991; 88:6264-8.

[18] Seabra MC, Goldstein JL, Sudhof TC, Brown MS. Rab geranylgeranyl transferases. A multisubunit enzyme that prenylates GTP-binding proteins terminating in Cys-XCys or Cys-Cys. J Biol Chem 1992; 267:14497-503. 
[19] Tanaka T, Tanaka M, Mitsui Y. Analysis of expressed sequence tags (ESTs) of the parasitic protozoa, Entamoeba histolytica. Biochem Biophys Res Commun 1997; 236:611-5.

[20] Rodriguez-Paris JM, Nolta KV, Steck TL. Characterization of lysosomes isolated from Dictyostelium discoideum by magnetic fractionation. J Biol Chem 1993; 268:9110-6.

[21] Diamond LS, Harlow DR, Cunnick CC. A new medium for the axenic culture of Entamoeba histolytica and other Entamoeba. Trans R Soc Trop Med Hyg 1978; 72:431-2.

[22] Fish WW. Rapid colorimetric micromethod for the quantitation of complexed iron in biological samples. Methods Enzymol 1988; 158:357-64.

[23] Temesvari LA, Rodriguez-Paris JM, Bush JM, Zhang L, Cardelli JA. Involvement of the vacuolar proton-translocating ATPase in multiple steps of the endo-lysosomal system and in the contractile vacuole system of Dictyostelium discoideum. J Cell Sci 1996; 109:1479-95.

[24] Temesvari L, Rodriguez-Paris J, Bush J, Steck TL, Cardelli J. Characterization of lysosomal membrane proteins of Dictyostelium discoideum. A complex population of acidic integral membrane glycoproteins, Rab GTP-binding proteins and vacuolar ATPase subunits. J Biol Chem 1994; 269:25719-27.

[25] Temesvari LA, Bush JM, Peterson MD, Novak KD, Titus MA, Cardelli JA. Examination of the endosomal and lysosomal pathways in Dictyostelium discoideum myosin I mutants. J Cell Sci 1996; 109:663-73.

[26] North MJ, Nicol K, Sands TW, Cotter DA. Acid-activatable cysteine proteinases in the cellular slime mold Dictyostelium discoideum. J Biol Chem 1996; 271:14462-7.

[27] Laemmli UK. Cleavage of structural proteins during the assembly of the head of bacteriophage T4. Nature 1970; 227:680-5.

[28] Tannich E, Horstmann RD, Knobloch J, Arnold HH. Genomic DNA differences between pathogenic and nonpathogenic Entamoeba histolytica. Proc Natl Acad Sci USA 1989; 86:5118-22.

[29] Wilbur WJ, Lipman DJ. Rapid similarity searches of nucleic acid and protein data banks. Proc Natl Acad Sci USA 1983; 80:726-30.

[30] Towbin H, Staehelin T, Gordon J. Electrophoretic transfer of proteins from polyacrylamide gels to nitrocellulose sheets: Procedure and some applications. Proc Natl Acad Sci USA 1979; 76:4350-4.
[31] Lohden U, Bakker-Grunwald T. Magnetic separation of pinocytic vesicles of defined age from Entamoeba histolytica. Anal Biochem 1989; 182:77-83.

[32] Ankri S, Stolarsky T, Mirelman D. Antisense inhibition of expression of cysteine proteinases does not affect Entamoeba histolytica cytopathic or haemolytic activity but inhibits phagocytosis. Mol Microbiol 1998; 28:777-85.

[33] de Meester F, Shaw E, Schoize H, Stolarsky T, Mirelman D. Specific labeling of cysteine proteinases in pathogenic and non-pathogenic Entamoeba histolytica. Infect Immun 1990; 58:1396-401.

[34] Claus V, Jahraus A, Tjelle T, Berg T, Kirschke H, Faulstich $\mathrm{H}$, Griffiths G. Lysosomal enzyme trafficking between phagosomes, endosomes, and lysosomes in J774 macrophages. J Biol Chem 1998; 273:9842-51.

[35] Becker I, Perez-Montfort R, Perez-Torres A, Rondan-Zarate A, Montfort I, Perez-Tamayo R. Entamoeba histolytica: Localization of a 30-kDa cysteine proteinase using a monoclonal antibody. Exp Parasitol 1996; 82:171-81.

[36] Lohia A, Samuelson J. Heterogeneity of Entamoeba histolytica rac genes encoding p21 $1^{\text {rac }}$ homologues. Gene 1996; 173:205-8.

[37] Pai EF, Kabsch W, Krengel U, Holmes KC, John J, Wittinghofer A. Structure of the guanine-nucleotide binding domain of the Ha ras oncogene product p21 in the triphosphate conformation. Nature 1989; 341:209-14.

[38] Urbe S, Huber LA, Zerial M, Tooze SA, Parton RG. Rab 11, a small GTPase associated with both constitutive and regulated secretory pathway in PC 12 cells. FEBS Lett 1993; 334:175-82.

[39] Ulrich O, Reinsch S, Urbe S, Zerial M, Parton RG. Rab 11 regulates recycling through the pericentriolar recycling endosome. J Cell Biol 1996; 135:913-24.

[40] Press B, Feng Y, Hoflack B, Wandinger-Ness A. Mutant Rab7 causes the accumulation of cathepsin D and cationindependent mannose 6-phosphate receptor in an early endocytic compartment. J Cell Biol 1998; 140:1075-89.

[41] Laurent O, Bruckert F, Adessi C, Satre M. In vitro reconstituted Dictyostelium discoideum early endosome fusion is regulated by Rab7 but proceeds in the absence of ATP$\mathrm{Mg}^{2+}$ from the bulk solution. J Biol Chem 1998; 273:793-9.

[42] Mukhopadhyay A, Funato K, Stahl PD. Rab7 regulates transport from early to late endocytic compartments in Xenopus oocytes. J Biol Chem 1997; 272:13055-9. 